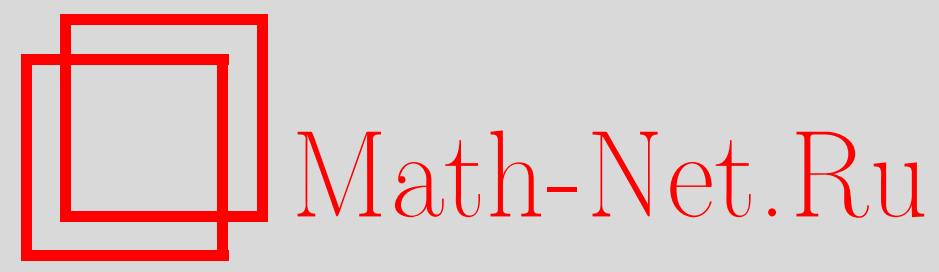

П. С. Рузанкин, Построение оптимального совместного распределения двух случайных величин, Теория вероятн. и ее примен., 2001, том 46, выпуск 2, 275-296

DOI: https://doi.org/10.4213/tvp3918

Использование Общероссийского математического портала MathNet.Ru подразумевает, что вы прочитали и согласны с пользовательским соглашением

http://www . mathnet.ru/rus/agreement

Параметры загрузки:

IP: 18.234 .197 .8

26 апреля 2023 г., 13:42:09

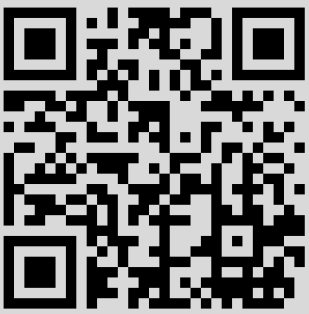




\title{
ПОСТРОЕНИЕ ОПТИМАЛЬНОГО СОВМЕСТНОГО РАСПРЕДЕЛЕНИЯ ДВУХ СЛУЧАЙНЫХ ВЕЛИЧИН ${ }^{1)}$
}

\begin{abstract}
Настоящая работа посвящена решению задачи Монжа-Канторовича для одного класса функционалов на вещественной оси. В качестве приложения получено усиление известного результата П. Майора, касающегося точности пуассоновской аппроксимации биномиального распределения.
\end{abstract}

Ключевые слова и фразы: задача Монжа-Канторовича, пуассоновская аппроксимация биномиального распределения.

1. Введение и формулировка основных результатов. Пусть на полном сепарабельном метрическом пространстве $(U, r)$ заданы две вероятностные борелевские меры $P$ и $Q$ и $M$ - пространство всех вероятностных борелевских мер $\mu$ на $U \times U$ с фиксированными маргиналами $\mu(\cdot \times U)=P(\cdot), \mu(U \times \cdot)=Q(\cdot)$. Задача Монжа-Канторовича (см. [1], [2] и обзор результатов в [3]) заключается в определении значения

$$
\kappa(P, Q)=\inf \left\{\int_{U \times U} f(v, w) \mu(d v, d w): \mu \in M\right\},
$$

где $f$ - заданная вещественнозначная функция, и в нахождении меры $\mu \in M$ (если таковая существует), на которой достигается эта нижняя грань. Частичное решение этой задачи дают так называемые теоремы двойственности, выражающие $\kappa(P, Q)$ в виде супремума некоторого функционала более простой структуры, определяемой только распределениями $P$ и $Q$ (см. [3]). Однако при этом не всегда ясен алгоритм вычисления этого супремума. Полное решение задачи МонжаКанторовича удается получить лишь в некоторых специальных случаях. Так, например, если $U=\mathbf{R}-$ вещественная прямая с естественной метрикой и $f(v, w)=g(v-w)$, где $g$ - выпуклая неотрицательная функция (см. [3]), то $\kappa(P, Q)=\int_{\mathbf{R}^{2}} g(v-w) d H(v, w)=$

${ }^{*}$ Институт математики им. С. Л. Соболева СО РАН, просп. Академика Коптюга, 4, 630090 Новосибирск, Россия; e-mail: ruzankin@math.nsc.ru

1) Работа выполнена при финансовой поддержке Российского фонда фундаментальных исследований, коды проектов 00-01-00802, 00-15-96178, и INTAS, код проекта 99-01317. 
$\int_{0}^{1} g\left(F^{-1}(u)-G^{-1}(u)\right) d u$, где $F$ и $G-$ функции распределения, соответствующие мерам $P$ и $Q, F^{-1}$ и $G^{-1}-$ квантильные преобразования $F$ и $G, H(v, w)=\min \{F(v), G(w)\}$. Для некоторых специальных классов распределений в $\mathbf{R}^{n}$ также получены полные решения этой задачи (см. [3]).

Мы будем рассматривать функционал $\kappa(P, Q)$ для ядер вида $f(v, w)=I(r(v, w)>z)$, где через $I$ обозначена индикаторная функция и $z$ - произвольная неотрицательная постоянная. В этом случае выражение (1) тождественно совпадает с величиной

$$
\rho(z, P, Q)=\inf _{\xi, \eta}\{\mathbf{P}\{r(\xi, \eta)>z\}\},
$$

где инфимум берется по всем случайным величинам $\xi$ и $\eta$, заданным на одном вероятностном пространстве, с распределениями $P$ и $Q$ соответственно. Определим также функционал

$$
\beta(z, P, Q)=\sup \left\{P(H)-Q\left(H^{z}\right): H \text { замкнуто в } U\right\},
$$

где $H^{z}=\{y \in U: r(H, y) \leqslant z\}$. Р. М. Дадли в [4] доказал теорему двойственности, связывающую введенные расстояния:

$$
\rho(z, P, Q)=\beta(z, P, Q) .
$$

Отметим, что при $z=0$ это соотношение при меньших ограничениях независимо доказал Р. Л. Добрушин в [5]. Напомним, что $\beta(0, P, Q)-$ это расстояние полной вариации между $P$ и $Q, a \inf \{z: \beta(z, P, Q) \leqslant z\}-$ расстояние Прохорова, метризуюшее слабую сходимость мер (см. [6]).

В [4] Р. М. Дадли также доказал, что нижняя грань в (2) всегда достигается, и построил оптимальное совместное рспределение с помощью дискретной аппроксимации. Для случая $U=\mathbf{R}$ более конструктивное построение указанного распределения изложено в п. 4. В отличие от (2), верхняя грань в (3) достигается не всегда.

3 а м е ч а н и е 1. На самом деле равенство (4) доказано в [4] для внутренне регулярных (например, полных) сепарабельных метрических пространств $U$. Другое доказательство (4) было предложено Г. Шэем в [7]. Однако в его доказательстве по существу используется не имеющая места непрерывность в топологии слабой сходимости функционала $\rho(z, P, Q)$ по аргументам $P$ и $Q$ (см. замечание 2$)$. Так что предложенное в [7] доказательство соотношения (4) неверно.

В работе [5] равенство (4) при $z=0$ доказано для произвольных сепарабельных (необязательно внутренне регулярных) метрических пространств $U$. Хотя в [5] отсутствует требование сепарабельности $U$, в несепарабельных метрических пространствах приведенное доказательство некорректно (см. замечание в [8]). 
Соотношение (4) является полезным для обоснования таких свойств функционала $\rho$, как непрерывность справа по $z$ (см. предложение 2), однако оно не дает нам способа вычисления значения этого функционала для конкретных распределений $P$ и $Q$. В случае, когда $U=\mathbf{R}$ - вещественная прямая с естественной метрикой и $z>0$, для вычисления значений функционала $\rho$ может быть полезна следующая

Теорема 1. Пусть $z>0$. Тогда

$$
\rho(z, P, Q)=\lim _{y \rightarrow \infty} S(y)-1=\lim _{y \rightarrow \infty} T(y)-1,
$$

где функции $S$ и тадаются соотношениями

$$
\begin{gathered}
\lim _{y \rightarrow-\infty} S(y)=\lim _{y \rightarrow-\infty} T(y)=0 \\
d S(y)=\max \{d F(y), T(y+d y-z)-S(y)\} \\
d T(y)=\max \{d G(y), S(y+d y-z)-T(y)\}
\end{gathered}
$$

при всех у и требованием непрерывности функций $S$ и $T$ слева. Функчии $S$ и $T$, удовлетворяющие перечисленным условиям, существуют и задаются этими условиями единственным образом.

Существование и единственность функций $S$ и $T$ устанавливаются в доказательстве предложения 1 . Выполнение $(6),(7)$ и условия непрерывности слева означает следующее:

$$
\begin{aligned}
S(y+w)= & S(y)+P([y, y+w)) \\
& +\sup _{0<v \leqslant w}(T(y+v-z)-S(y)-P([y, y+v)))^{+} \\
T(y+w)= & T(y)+Q([y, y+w)) \\
& +\sup _{0<v \leqslant w}(S(y+v-z)-T(y)-Q([y, y+v)))^{+}
\end{aligned}
$$

при всех $y$ и $w>0$. Символом $(\cdot)^{+}$обозначена положительная часть числа, т.е. $(\cdot)^{+}=\max \{\cdot, 0\}$. Так как функционал $\rho(z, P, Q)$ непрерывен справа по $z$ (см. предложение 2), то с помощью теоремы 1 можно доопределить значение этого функционала и при $z=0$.

Предложение 1. Oпределенные в теореме 1 бунжции $S$ и $T$ могут быть заданы следугшим образом:

$$
S(y)=\lim _{L \rightarrow-\infty} S_{L}(y), \quad T(y)=\lim _{L \rightarrow-\infty} T_{L}(y) \quad \text { nрu всех } y
$$

где функиии $S_{L}$ и $T_{L}$ определяются единственным образом следуюиими соотношениями:

$$
S_{L}(y)=T_{L}(y)=0 \quad n p u \quad y \leqslant L
$$




$$
\begin{aligned}
S_{L}(y+w)= & S_{L}(y)+P([y, y+w)) \\
& +\sup _{0<v \leqslant w}\left(T_{L}(y+v-z)-S_{L}(y)-P([y, y+v))\right)^{+}, \\
T_{L}(y+w)= & T_{L}(y)+Q([y, y+w)) \\
& +\sup _{0<v \leqslant w}\left(S_{L}(y+v-z)-T_{L}(y)-Q([y, y+v))\right)^{+}
\end{aligned}
$$

при любых вещественных $y \geqslant L u w>0$. При этом выполнено

$$
\lim _{L \rightarrow-\infty} \lim _{y \rightarrow \infty} S_{L}(y)=\lim _{y \rightarrow \infty} S(y)=\lim _{L \rightarrow-\infty} \lim _{y \rightarrow \infty} T_{L}(y)=\lim _{y \rightarrow \infty} T(y) .
$$

Соотношения (11)-(13) дают нам возможность вычислить значения функций $S_{L}$ и $T_{L}$. Действительно, при $y \leqslant L$ значения $S_{L}(y)$ и $T_{L}(y)$ заданы. Из соотношений $(12),(13)$ получим значения $S_{L}(y)$ и $T_{L}(y)$ при $L<y \leqslant L+z$. Теперь можно вычислить значения $S_{L}(y)$ и $T_{L}(y)$ при $L+z<y \leqslant L+2 z, L+2 z<y \leqslant L+3 z, \ldots$. Таким образом можно вычислить $S_{L}(y)$ и $T_{L}(y)$ при всех $y$.

Заметим, что если для некоторого $K$ выполнено $F(K)=G(K)=0$, то $S_{L}(y) \equiv S_{K}(y)$ и $T_{L}(y) \equiv T_{K}(y)$ при всех $L \leqslant K$. А значит, выполнено следуюшее

Следствие. Пусть $z>0$ u $F(K)=G(K)=0$ для некоторого $K$. Тогда функии $S$ и $Т$ в теореме 1 можно задать следующим образом:

$$
\begin{aligned}
S(y)= & T(y)=0 \quad \text { nрu } y \leqslant K \\
S(y+w)= & S(y)+P([y, y+w)) \\
& +\sup _{0<v \leqslant w}(T(y+v-z)-S(y)-P([y, y+v)))^{+} \\
T(y+w)= & T(y)+Q([y, y+w)) \\
& +\sup _{0<v \leqslant w}(S(y+v-z)-T(y)-Q([y, y+v)))^{+}
\end{aligned}
$$

при любых вещественных $y \geqslant K u w>0$.

Некоторые примеры применения теоремы 1 помещены в п. 3. Важным приложением этой теоремы является получение оценки близости биномиального и соответствующего пуассоновского законов. Эта оценка без доказательства помещена в п. 5. Ее доказательство будет опубликовано в отдельной статье.

Следующая теорема позволяет применить следствие для симметричных распределений.

Теорема 2. Пусть распределения $P$ u симметричны. Зададим тажже распределения $\breve{P}$ и $\breve{Q}$ равенствами

$\breve{P}(A)=P(A \cap\{0\})+2 P(A \cap(0, \infty)), \quad \breve{Q}(A)=Q(A \cap\{0\})+2 Q(A \cap(0, \infty))$

для всех борелевских $A \subset \mathbf{R}$. Тогда $\rho(z, P, Q)=\rho(z, \breve{P}, \breve{Q})$ nри всех $z \geqslant 0$. 
Для распределений на множестве неотрицательных целых чисел следствие можно переформулировать следующим образом.

Теорема 3. Пусть $P$ и - произвольные распределения на множестве неотричательных челых чисел и $z \geqslant 0$ челое. Тогда

$$
\begin{aligned}
\rho(z, P, Q) & =\lim _{j \rightarrow \infty} S_{j}-1=\lim _{j \rightarrow \infty} T_{j}-1 \\
& =\sum_{j=0}^{\infty}\left(S_{j}-T_{j+z}\right)^{+}=\sum_{j=0}^{\infty}\left(T_{j}-S_{j+z}\right)^{+}
\end{aligned}
$$

$2 \partial e$

$$
\begin{aligned}
& S_{j}=T_{j}=0 \quad \text { nрu всех } j<0 \\
& S_{j}=P(\{j\})+\max \left\{S_{j-1}, T_{j-1-z}\right\} \\
& T_{j}=Q(\{j\})+\max \left\{T_{j-1}, S_{j-1-z}\right\}
\end{aligned}
$$

при всех $j \geqslant 0$.

Автор выражает искреннюю благодарность И.С. Борисову за постановку задачи и полезные замечания.

2. Доказательство результатов. Всюду в дальнейшем для любого вещественного числа $y$ символом $\lfloor y\rfloor$ будем обозначать наибольшее целое число, не превосходящее $y$, а символом $\lceil y\rceil$ - наименьшее целое, не меньшее $y$. Очевидно, $\lceil y\rceil=-\lfloor-y\rfloor$.

Предложение 2. Для любых борелевских вероятностных мер $P$ u $Q$ на польском пространстве $U$ функчионал $\rho(z, P, Q)$ непрерывен справа по $z$.

Д ок а з а т льст в о. Выберем произвольно $\varepsilon>0$. В силу равенства (4) существует такое замкнутое $H \subset U$, что $P(H)-Q\left(H^{z}\right)>$ $\rho(z, P, Q)-\varepsilon / 2$. Выберем такое $\delta>0$, что $Q\left(H^{z+\delta}\right)-Q\left(H^{z}\right)<\varepsilon / 2$. Тогда для каждого $u, 0<u<\delta$, выполнено

$$
\rho(z+u, P, Q) \geqslant P(H)-Q\left(H^{z+u}\right)>P(H)-Q\left(H^{z}\right)-\frac{\varepsilon}{2}>\rho(z, P, Q)-\varepsilon .
$$

Таким образом, для любого $\varepsilon>0$ существует такое $\delta>0$, что для всех $u, 0 \leqslant u<\delta$, выполнено $\rho(z+u, P, Q)>\rho(z, P, Q)-\varepsilon$.

Доказательст в о те оремы 3 . Фиксируем целое $z \geqslant 0$. Обозначим $p_{j}=P(\{j\}), q_{j}=Q(\{j\}), j=0,1,2, \ldots$

Пусть заданы неотрицательные числа $a_{0}, a_{1}, a_{2}, \ldots, b_{0}, b_{1}, b_{2}, \ldots$ Обозначим через $H(\bar{a}, \bar{b})$ множество всех наборов чисел $\bar{x}=\left\{x_{i j}\right\}_{i j}$, удовлетворяющих следующим условиям: $x_{i j} \geqslant 0$ для всех $i$ и $j, \sum_{j=i-z}^{i+z} x_{i j} \leqslant a_{i}$ для всех $i \geqslant 0, \sum_{i=j-z}^{j+z} x_{i j} \leqslant b_{j}$ для всех $j \geqslant 0, x_{i j}=0$, если $i<0$ или $j<0$. 
Для набора чисел $\bar{x} \in H(\bar{a}, \bar{b})$ обозначим $\delta(\bar{x}, \bar{a})=\sum_{i=0}^{\infty}\left(a_{i}-\sum_{j=i-z}^{i+z} x_{i j}\right)$. Имеем

$$
\rho(z, P, Q)=R_{z}\left(p_{0}, p_{1}, \ldots ; q_{0}, q_{1}, \ldots\right),
$$

где

$$
R_{z}\left(a_{0}, a_{1}, \ldots ; b_{0}, b_{1}, \ldots\right)=\inf \{\delta(\bar{x}, \bar{a}): \bar{x} \in H(\bar{a}, \bar{b})\} .
$$

Далее нам потребуются следующие легко проверяемые тождества, имеющие место при всех неотрицательных $u, v, w$ :

$$
\begin{aligned}
(u-v)^{+} & =u-\min \{u, v\} \\
(u-(v+w))^{+} & =\left((u-v)^{+}-w\right)^{+} \\
\left(u-(v-w)^{+}\right)^{+} & =(u+w-\max \{v, w\})^{+} \\
\left((u-w)^{+}-(v-w)^{+}\right)^{+} & =(u-\max \{v, w\})^{+}
\end{aligned}
$$

Далее мы покажем, что

$$
\begin{aligned}
& \inf \{\delta(\bar{x}, \bar{a}): \bar{x} \in H(\bar{a}, \bar{b})\} \\
& =\inf \left\{\delta(\bar{x}, \bar{a}): \bar{x} \in H(\bar{a}, \bar{b}), x_{00}=s_{00}, x_{01}=s_{01}, \ldots,\right. \\
& \left.\quad x_{0 z}=s_{0 z}, x_{10}=s_{10}, x_{20}=s_{20}, \ldots, x_{z 0}=s_{z 0}\right\},
\end{aligned}
$$

где

$$
\begin{aligned}
s_{00}= & \min \left\{a_{0}, b_{0}\right\} \\
s_{01}= & \min \left\{b_{1}, a_{0}-s_{00}\right\}=\min \left\{b_{1},\left(a_{0}-b_{0}\right)^{+}\right\} \\
s_{02}= & \min \left\{b_{2}, a_{0}-s_{00}-s_{01}\right\}=\min \left\{b_{2},\left(a_{0}-\left(b_{0}+b_{1}\right)\right)^{+}\right\} \\
& \cdots \\
s_{0 z}= & \min \left\{b_{z}, a_{0}-s_{00}-s_{01}-\cdots-s_{0, z-1}\right\} \\
= & \min \left\{b_{z},\left(a_{0}-\left(b_{0}+b_{1}+\cdots+b_{z-1}\right)\right)^{+}\right\} \\
s_{10}= & \min \left\{a_{1}, b_{0}-s_{00}\right\}=\min \left\{a_{1},\left(b_{0}-a_{0}\right)^{+}\right\} \\
& \cdots \\
s_{z 0}= & \min \left\{a_{z}, b_{0}-s_{00}-s_{10}-\cdots-s_{z-1,0}\right\} \\
= & \min \left\{a_{z},\left(b_{0}-\left(a_{0}+a_{1}+\cdots+a_{z-1}\right)\right)^{+}\right\}
\end{aligned}
$$

Имея равенство (29), можно выделить первое слагаемое из каждой суммы $\delta(\bar{x}, \bar{a})$, стоящей в выражении $\inf \{\delta(\bar{x}, \bar{a}): \bar{x} \in H(\bar{a}, \bar{b})\}$. Получим

$$
\begin{gathered}
R_{z}\left(a_{0}, a_{1}, \ldots ; b_{0}, b_{1}, \ldots\right)=\inf \{\delta(\bar{x}, \bar{a}): \bar{x} \in H(\bar{a}, \bar{b})\} \\
=\inf \left\{\delta(\bar{x}, \bar{a}): \bar{x} \in H(\bar{a}, \bar{b}), x_{00}=s_{00}, \ldots,\right. \\
\left.\quad x_{0 z}=s_{0 z}, x_{10}=s_{10}, \ldots, x_{z 0}=s_{z 0}\right\} \\
=\left(a_{0}-s_{00}-s_{01}-\cdots-s_{0 z}\right)
\end{gathered}
$$




$$
\begin{aligned}
+ & R_{z}\left(a_{1}-s_{10}, a_{2}-s_{20}, \ldots, a_{z}-s_{z 0}, a_{z+1}, a_{z+2}, a_{z+3}, \ldots ;\right. \\
& \left.b_{1}-s_{01}, b_{2}-s_{02}, \ldots, b_{z}-s_{0 z}, b_{z+1}, b_{z+2}, b_{z+3}, \ldots\right) \\
=\left(a_{0}-\left(b_{0}+b_{1}+\cdots+b_{z}\right)\right)^{+} & +R_{z}\left(\left(a_{1}-\left(b_{0}-a_{0}\right)^{+}\right)^{+},\left(a_{2}-\left(b_{0}-\left(a_{0}+a_{1}\right)\right)^{+}\right)^{+}, \ldots,\right. \\
& \left(a_{z}-\left(b_{0}-\left(a_{0}+a_{1}+\cdots+a_{z-1}\right)\right)^{+}\right)^{+}, a_{z+1}, a_{z+2}, a_{z+3}, \ldots ; \\
& \left(b_{1}-\left(a_{0}-b_{0}\right)^{+}\right)^{+},\left(b_{2}-\left(a_{0}-\left(b_{0}+b_{1}\right)\right)^{+}\right)^{+}, \ldots, \\
& \left.\left(b_{z}-\left(a_{0}-\left(b_{0}+b_{1}+\cdots+b_{z-1}\right)\right)^{+}\right)^{+}, b_{z+1}, b_{z+2}, b_{z+3}, \ldots\right) .
\end{aligned}
$$

\section{В силу равенства}

$$
\begin{aligned}
& R_{z}\left(a_{0}, a_{1}, \ldots ; b_{0}, b_{1}, \ldots\right)=\left(a_{0}-\left(b_{0}+b_{1}+\cdots+b_{z}\right)\right)^{+} \\
& +R_{z}\left(\left(a_{1}-\left(b_{0}-a_{0}\right)^{+}\right)^{+},\left(a_{2}-\left(b_{0}-\left(a_{0}+a_{1}\right)\right)^{+}\right)^{+}, \ldots,\right. \\
& \left(a_{z}-\left(b_{0}-\left(a_{0}+a_{1}+\cdots+a_{z-1}\right)\right)^{+}\right)^{+}, a_{z+1}, a_{z+2}, a_{z+3}, \ldots ; \\
& \left(b_{1}-\left(a_{0}-b_{0}\right)^{+}\right)^{+},\left(b_{2}-\left(a_{0}-\left(b_{0}+b_{1}\right)\right)^{+}\right)^{+}, \ldots, \\
& \left.\left(b_{z}-\left(a_{0}-\left(b_{0}+b_{1}+\cdots+b_{z-1}\right)\right)^{+}\right)^{+}, b_{z+1}, b_{z+2}, b_{z+3}, \ldots\right),
\end{aligned}
$$

тождеств $(25)-(28)$ и неравенств $S_{0} \leqslant S_{1} \leqslant S_{2} \leqslant \cdots, T_{0} \leqslant T_{1} \leqslant T_{2} \leqslant \cdots$ несложно показать по индукции, что для каждого $k=0,1,2, \ldots$ выполняется соотношение

$$
\begin{aligned}
& R_{z}\left(p_{0}, p_{1}, \ldots ; q_{0}, q_{1}, \ldots\right)=\sum_{j=0}^{k-1}\left(S_{j}-T_{j+z}\right)^{+} \\
&+R_{z}(\left(S_{k}-\max \left\{S_{k-1}, T_{k-1}\right\}\right)^{+},\left(S_{k+1}-\max \left\{S_{k}, T_{k-1}\right\}\right)^{+}, \ldots, \\
&\left(S_{k+z-1}-\max \left\{S_{k+z-2}, T_{k-1}\right\}\right)^{+}, p_{k+z}, p_{k+z+1}, p_{k+z+2}, \ldots ; \\
&\left(T_{k}-\max \left\{T_{k-1}, S_{k-1}\right\}\right)^{+},\left(T_{k+1}-\max \left\{T_{k}, S_{k-1}\right\}\right)^{+}, \ldots, \\
&\left.\left(T_{k+z-1}-\max \left\{T_{k+z-2}, S_{k-1}\right\}\right)^{+}, q_{k+z}, q_{k+z+1}, q_{k+z+2}, \ldots\right) .
\end{aligned}
$$

Таким образом,

$$
R_{z}\left(p_{0}, p_{1}, \ldots ; q_{0}, q_{1}, \ldots\right)=\sum_{j=0}^{k-1}\left(S_{j}-T_{j+z}\right)^{+}+h(k),
$$

где $0 \leqslant h(k) \leqslant \sum_{j=k}^{\infty} p_{j}$. Устремляя $k \rightarrow \infty$, получим равенство

$$
R_{z}\left(p_{0}, p_{1}, \ldots ; q_{0}, q_{1}, \ldots\right)=\sum_{j=0}^{\infty}\left(S_{j}-T_{j+z}\right)^{+}
$$

Теперь перепишем последнее равенство в другом виде: $R_{z}\left(p_{0}, p_{1}, \ldots\right.$; $\left.q_{0}, q_{1}, \ldots\right)=\sum_{j=0}^{\infty}\left(S_{j}-T_{j+z}\right)^{+}=\sum_{j=z}^{\infty}\left(T_{j+1}-\left(T_{j}+q_{j}\right)\right)=\sum_{j=0}^{\infty}\left(T_{j+1}-\right.$ 
$\left.\left(T_{j}+q_{j}\right)\right)=\lim _{j \rightarrow \infty} T_{j}-\sum_{j=0}^{\infty} q_{j}=\lim _{j \rightarrow \infty} T_{j}-1$. Очевидно также, что $R_{z}\left(p_{0}, p_{1}, \ldots ; q_{0}, q_{1}, \ldots\right)=R_{z}\left(q_{0}, q_{1}, \ldots ; p_{0}, p_{1}, \ldots\right)=\sum_{j=0}^{\infty}\left(T_{j}-S_{j+z}\right)^{+}=$ $\lim _{j \rightarrow \infty} S_{j}-1$.

Таким образом, для завершения доказательства теоремы осталось установить справедливость равенства (29). Для этого сначала покажем, что при каждом $l, 0 \leqslant l \leqslant z$, выполнено

$$
\begin{gathered}
\inf \left\{\delta(\bar{x}, \bar{a}): \bar{x} \in H(\bar{a}, \bar{b}), x_{00}=s_{00}, x_{01}=s_{01}, \ldots, x_{0, l-1}=s_{0, l-1}\right\} \\
=\inf \left\{\delta(\bar{x}, \bar{a}): \bar{x} \in H(\bar{a}, \bar{b}), x_{00}=s_{00}, x_{01}=s_{01}, \ldots,\right. \\
\left.x_{0, l-1}=s_{0, l-1}, x_{0 l}=s_{0 l}\right\} .
\end{gathered}
$$

Чтобы убедиться в последнем, по любому набору $\bar{x}=\left\{x_{i j}\right\}_{i j}$ из $H(\bar{a}, \bar{b})$, удовлетворяюшему условиям $x_{00}=s_{00}, x_{01}=s_{01}, \ldots, x_{0, l-1}=s_{0, l-1}$, построим набор $\bar{y}=\left\{y_{i j}\right\}_{i j}$ из класса $H(\bar{a}, \bar{b})$, для которого $y_{00}=$ $s_{00}, \ldots, y_{0, l-1}=s_{0, l-1}, y_{0 l}=s_{0 l}$ и $\delta(\bar{x}, \bar{a}) \geqslant \delta(\bar{y}, \bar{a})$. Пусть задан такой набор $\left\{x_{i j}\right\}_{i j}$. Из определения $s_{00}, s_{01}, \ldots, s_{0, l-1}$ и из принадлежности $\bar{x} \in H(\bar{a}, \bar{b})$ следует, что $x_{0 l} \leqslant s_{0 l}$. Положим $y_{i j}=x_{i j}$ при выполнении одного из четырех условий: $i\langle 0, i\rangle l+z, j\langle l, j\rangle z$. Зададим $y_{0 l}=s_{0 l}$. Подберем $y_{0, l+1}, y_{0, l+2}, \ldots, y_{0 z}$ так, чтобы выполнялись следующие неравенства:

$$
\begin{gathered}
0 \leqslant y_{0, l+1} \leqslant x_{0, l+1}, \ldots, 0 \leqslant y_{0 z} \leqslant x_{0 z}, \\
\left(x_{0, l+1}+\cdots+x_{0 z}\right)-\left(y_{0, l+1}+\cdots+y_{0 z}\right) \leqslant y_{0 l}-x_{0 l}, \quad y_{01}+\cdots+y_{0 z} \leqslant a_{0} .
\end{gathered}
$$

Также подберем такие $y_{1, l}, y_{2, l}, \ldots, y_{l+z, l}$, что

$$
\begin{gathered}
0 \leqslant y_{1, l} \leqslant x_{1, l}, \ldots, 0 \leqslant y_{l+z, l} \leqslant x_{l+z, l}, \\
\left(x_{1, l}+\cdots+x_{l+z, l}\right)-\left(y_{1, l}+\cdots+y_{z+l, l}\right) \leqslant y_{0 l}-x_{0 l}, \quad y_{0 l}+\cdots+y_{l+z, l} \leqslant b_{l} .
\end{gathered}
$$

Затем для $1 \leqslant i \leqslant l+z, l+1 \leqslant j \leqslant z$ подберем $y_{i j} \geqslant x_{i j}$ так, чтобы набор $\bar{y}$ принадлежал $H(\bar{a}, \bar{b})$ и

$\sum_{1 \leqslant i \leqslant l+z, l+1 \leqslant j \leqslant z}\left(y_{i j}-x_{i j}\right)=\min \left\{\left(x_{0, l+1}+\cdots+x_{0 z}\right)-\left(y_{0, l+1}+\cdots+y_{0 z}\right)\right.$,

$$
\left.\left(x_{1, l}+\cdots+x_{l+z, l}\right)-\left(y_{1, l}+\cdots+y_{z+l, l}\right)\right\} .
$$

Тогда для построенного так набора $\left\{y_{i j}\right\}_{i j}$ получим

$$
\begin{aligned}
& \delta(\bar{x}, \bar{a})-\delta(\bar{y}, \bar{a})=\left(x_{0, l+1}+\cdots+x_{0 z}\right)-\left(y_{0, l+1}+\cdots+y_{0 z}\right) \\
&+\left(x_{1, l}+\cdots+x_{l+z, l}\right)-\left(y_{1, l}+\cdots+y_{z+l, l}\right)-\left(y_{0 l}-x_{0 l}\right) \\
&-\min \left\{\left(x_{0, l+1}+\cdots+x_{0 z}\right)-\left(y_{0, l+1}+\cdots+y_{0 z}\right),\right. \\
&\left.\left(x_{1, l}+\cdots+x_{l+z, l}\right)-\left(y_{1, l}+\cdots+y_{z+l, l}\right)\right\} \geqslant 0,
\end{aligned}
$$

что и требовалось. 
Tогда

$$
\begin{aligned}
& \inf \{\delta(\bar{x}, \bar{a}): \bar{x} \in H(\bar{a}, \bar{b})\}=\inf \left\{\delta(\bar{x}, \bar{a}): \bar{x} \in H(\bar{a}, \bar{b}), x_{00}=s_{00}\right\} \\
& \quad=\cdots=\inf \left\{\delta(\bar{x}, \bar{a}): \bar{x} \in H(\bar{a}, \bar{b}), x_{00}=s_{00}, x_{01}=s_{01}, \ldots, x_{0 z}=s_{0 z}\right\} .
\end{aligned}
$$

Проводя аналогичные рассуждения, можно показать, что

$$
\begin{gathered}
\inf \left\{\delta(\bar{x}, \bar{a}): \bar{x} \in H(\bar{a}, \bar{b}), x_{00}=s_{00}, \ldots, x_{0 z}=s_{0 z}\right\} \\
=\inf \left\{\delta(\bar{x}, \bar{a}): \bar{x} \in H(\bar{a}, \bar{b}), x_{00}=s_{00}, \ldots, x_{0 z}=s_{0 z}, x_{10}=s_{10}\right\} \\
=\cdots=\inf \left\{\delta(\bar{x}, \bar{a}): \bar{x} \in H(\bar{a}, \bar{b}), x_{00}=s_{00}, \ldots, x_{0 z}=s_{0 z},\right. \\
\left.x_{10}=s_{10}, x_{20}=s_{20}, \ldots, x_{z 0}=s_{z 0}\right\} .
\end{gathered}
$$

Таким образом, соотношение (29), а вместе с ним и теорема 3 доказаны.

Доказательст во п редложения 1. Для доказательства этого предложения необходимо также убедиться в сушествовании и единственности функций $S$ и $T$, удовлетворяющих соотношениям (5), (8), (9), что и будет установлено в конце доказательства. Фиксируем $L>-\infty$ и построим функции $S_{L}$ и $T_{L}$, удовлетворяющие соотношениям (11)-(13). Обозначим

$$
\begin{aligned}
\widetilde{S}_{L}(y, w)= & S_{L}(y)+P([y, y+w)) \\
& +\sup _{0<v \leqslant w}\left(T_{L}(y+v-z)-S_{L}(y)-P([y, y+v))\right)^{+}, \\
\tilde{T}_{L}(y, w)= & T_{L}(y)+Q([y, y+w)) \\
& +\sup _{0<v \leqslant w}\left(S_{L}(y+v-z)-T_{L}(y)-Q([y, y+v))\right)^{+} .
\end{aligned}
$$

Положим $S_{L}(y)=T_{L}(y)=0$ для всех $y \leqslant L$. Далее, последовательно для каждого $j=0,1,2, \ldots$ будем полагать $S_{L}(j z+w)=\widetilde{S}_{L}(j z, w), T_{L}(j z+$ $w)=\widetilde{T}_{L}(j z, w)$ при всех $w, 0<w \leqslant z$. Таким образом, функции $S_{L}$ и $T_{L}$ будут однозначно определены, и равенства (11) будут выполнены.

Докажем, что эти функции будут удовлетворять соотношениям (12), (13). Возьмем произвольно $y \geqslant L$ и набор положительных чисел $w_{1}, w_{2}, \ldots, w_{n}$. Несложно проверить, что если для каждого $j=1,2, \ldots, n$ выполнено

$$
S_{L}\left(y+w_{1}+w_{2}+\cdots+w_{j-1}+w\right)=\widetilde{S}_{L}\left(y+w_{1}+w_{2}+\cdots+w_{j-1}, w\right)
$$

при всех $w, 0<w<w_{j}$, то

$$
\begin{aligned}
& S_{L}(y+w)=\widetilde{S}_{L}(y, w) \quad \text { при всех } 0<w<w_{1}+w_{2}+\cdots+w_{n}, \\
& S_{L}\left(y+w_{0}+w\right)=\widetilde{S}_{L}\left(y+w_{0}, w\right) \\
& \quad \text { при всех } 0<w_{0}<w_{1}, 0<w<w_{1}+w_{2}+\cdots+w_{n}-w_{0} .
\end{aligned}
$$


Соотношение (30) доказывает (12) при всех $y=L+j z, j=1,2,3, \ldots$, а соотношение (31) - при всех $y \neq L+j z, j=1,2,3, \ldots$ (нужно в (12) подставить $y+w_{0}$ вместо $y$ ). Аналогично доказывается и (13).

Пусть $f$ и $g$ - произвольные функции, удовлетворяющие условиям: $0 \leqslant f(y) \leqslant \varepsilon, 0 \leqslant g(y) \leqslant \varepsilon$ при всех $y$. Определим функции $S_{l}^{\prime}$ и $T_{L}^{\prime}$ равенствами

$$
S_{L}^{\prime}(y)=f(y), \quad T_{L}^{\prime}(y)=g(y) \quad \text { при всех } y \leqslant L
$$

и соотношениями (12), (13). Аналогично, функции $S_{L}^{\prime}$ и $T_{L}^{\prime}$ существуют и определены единственным образом. Индукцией по $j$ несложно показать, что для каждого $j=0,1,2, \ldots$ выполнено

$$
S_{L}(y) \leqslant S_{L}^{\prime}(y) \leqslant S_{L}(y)+\varepsilon, \quad T_{L}(y) \leqslant T_{L}^{\prime}(y) \leqslant T_{L}(y)+\varepsilon
$$

при всех $y, j z<y \leqslant(j+1) z$. А значит, последние неравенства выполнены для всех $y$. Имеем также при всех $M \leqslant L$

$$
\max \left\{S_{M}(y), T_{M}(y)\right\} \leqslant P((-\infty, L))+Q((-\infty, L)) \quad \text { при всех } y \leqslant L .
$$

Следовательно, при всех $M \leqslant L$ ввиду (32) будем иметь

$$
\begin{aligned}
& \max \left\{\left|S_{M}(y)-S_{L}(y)\right|,\left|T_{M}(y)-T_{L}(y)\right|\right\} \\
& \quad \leqslant P((-\infty, L))+Q((-\infty, L)) \text { при всех } y
\end{aligned}
$$

Значит, функции $S_{L}$ и $T_{L}$ равномерно сходятся при $L \rightarrow-\infty$ к некоторым предельным функциям $S$ и $T$. В силу равномерной сходимости $S$ и $T$ будут удовлетворять соотношениям (5), (8), (9) и (10), (14).

Таким образом, доказано существование функций $S$ и $T$, удовлетворяющих соотношениям (5)-(7). Докажем теперь, что эти соотношения задают функции $S$ и $T$ единственным образом. Предположим, что некоторые функции $S^{\prime}$ и $T^{\prime}$ также удовлетворяют соотношениям (5)-(7). Заметим, что ввиду (8) и (9) функции $S^{\prime}$ и $T^{\prime}$ возрастают. Фиксируем $\varepsilon>0$. Выберем такое $L>-\infty$, что $S^{\prime}(L)<\varepsilon / 2, T^{\prime}(L)<\varepsilon / 2$, $P((-\infty, L))+Q((-\infty, 0)<\varepsilon / 2$. Тогда при всех $y$ будут выполнены неравенства

$$
\begin{aligned}
\left|S^{\prime}(y)-S_{L}(y)\right|<\frac{\varepsilon}{2}, & \left|S(y)-S_{L}(y)\right|<\frac{\varepsilon}{2} \\
\left|T^{\prime}(y)-T_{L}(y)\right|<\frac{\varepsilon}{2}, & \left|T(y)-T_{L}(y)\right|<\frac{\varepsilon}{2} .
\end{aligned}
$$

Таким образом, для любого $\varepsilon>0$ выполнено

$$
\left|S^{\prime}(y)-S(y)\right|<\varepsilon, \quad\left|T^{\prime}(y)-T(y)\right|<\varepsilon \quad \text { при всех } y .
$$

Это и доказывает единственность функций $S$ и $T$, задаваемых соотношениями (5)-(7). 
Д ок азат ель с т в о сле д с т в и я. Корректность определения функций $S(y)$ и $T(y)$ не вызывает сомнений. Без ограничения общности можно считать, что $K=0$.

Рассмотрим сначала случай, когда распределения $P$ и $Q$ с целочисленными носителями, а $z \geqslant 1$ - целое. Преобразуем формулу (18) теоремы 3 . Обозначим $S_{j}^{\prime}=S_{j}-p_{j}, T_{j}^{\prime}=T_{j}-q_{j}$. Понятно, что

$$
\lim _{j \rightarrow \infty} S_{j}^{\prime}=\lim _{j \rightarrow \infty} S_{j}, \quad \lim _{j \rightarrow \infty} T_{j}^{\prime}=\lim _{j \rightarrow \infty} T_{j} .
$$

Подставив $S_{j}^{\prime}$ и $T_{j}^{\prime}$ в соотношения (20)-(22), получим

$$
\begin{aligned}
& S_{j}^{\prime}=T_{j}^{\prime}=0 \text { при } j \leqslant 0, \\
& S_{j}^{\prime}=\max \left\{S_{j-1}^{\prime}+p_{j-1}, T_{j-z-1}^{\prime}+q_{j-z-1}\right\} \quad \text { при } j>0, \\
& T_{j}^{\prime}=\max \left\{T_{j-1}^{\prime}+q_{j-1}, S_{j-z-1}^{\prime}+p_{j-z-1}\right\} \quad \text { при } j>0,
\end{aligned}
$$

где два последних равенства эквивалентны следующим соотношениям:

$$
\begin{array}{ll}
S_{j}^{\prime}=\max \left\{S_{j-1}^{\prime}+p_{j-1}, T_{j-z}^{\prime}\right\}, & j>0, \\
T_{j}^{\prime}=\max \left\{T_{j-1}^{\prime}+q_{j-1}, S_{j-z}^{\prime}\right\}, & j>0,
\end{array}
$$

поскольку $S_{j-1}^{\prime} \geqslant S_{j-2 z-1}^{\prime}+p_{j-2 z-1}, T_{j-1}^{\prime} \geqslant T_{j-2 z-1}^{\prime}+q_{j-2 z-1}$.

Далее, непосредственно из (34), (35) получим для целых $t \geqslant 1$ и $j \geqslant 0$

$$
S_{j+t}^{\prime}=\max \left\{S_{j}^{\prime}+p_{j}+p_{j+1}+\cdots+p_{j+t-1}, \max _{1 \leqslant l \leqslant t}\left\{T_{j+l-z}^{\prime}+\sum_{k=l}^{t-1} p_{j+k}\right\}\right\} .
$$

Следовательно, при $j \geqslant 0$ и $t \geqslant 1$

$$
S_{j+t}^{\prime}=S_{j}^{\prime}+\sum_{k=0}^{t-1} p_{j+k}+\max _{0 \leqslant l \leqslant t}\left(T_{j+l-z}^{\prime}-S_{j}^{\prime}-\sum_{k=0}^{l-1} p_{j+k}\right)^{+} .
$$

В (36) максимум можно брать по $0 \leqslant l \leqslant t$ вместо $1 \leqslant l \leqslant t$, так как $S_{j}^{\prime} \geqslant T_{j-z}$ при всех $j$. Аналогично,

$$
T_{j+t}^{\prime}=T_{j}^{\prime}+\sum_{k=0}^{t-1} q_{j+k}+\max _{0 \leqslant l \leqslant t}\left(S_{j+l-z}^{\prime}-T_{j}^{\prime}-\sum_{k=0}^{l-1} q_{j+k}\right)^{+} .
$$

Сравнив последние два выражения с (16), (17), видим, что $S(j)=S_{j}^{\prime}$, $T(j)=T_{j}^{\prime}$ при всех целых $j$, что и доказывает ввиду (33) утверждение следствия для целочисленных, а значит, и для произвольных неотрицательных заданных на одной решетке случайных величин и $z$, не меньшего, чем шаг решетки.

Докажем теперь утверждение следствия для произвольных распределений $P$ и $Q$ на множестве неотрицательных чисел. Фиксируем $z>0$. 
Обозначим $\gamma_{n}=z \cdot 2^{-n}, n=1,2,3, \ldots$ Для произвольной случайной величины $\varphi$ через $\hat{\varphi}_{n}$ обозначим

$$
\widehat{\varphi}_{n}=\left\lfloor\frac{\varphi}{\gamma_{n}}\right\rfloor \gamma_{n}, \quad n=1,2, \ldots
$$

Тогда для любых случайных величин $\varphi, \psi$ имеем $\mathbf{P}\left\{\left|\widehat{\varphi}_{n}-\widehat{\psi}_{n}\right|>j \gamma_{n}\right\} \leqslant$ $\mathbf{P}\left\{|\varphi-\psi|>j \gamma_{n}\right\}$ при всех целых $j$. А значит, выполнены неравенства

$$
\mathbf{P}\left\{|\varphi-\psi|>z+\gamma_{n}\right\} \leqslant \mathbf{P}\left\{\left|\widehat{\varphi}_{n}-\widehat{\psi}_{n}\right|>z\right\} \leqslant \mathbf{P}\{|\varphi-\psi|>z\} .
$$

Обозначим через $\widehat{P}_{n}, \widehat{Q}_{n}$ распределения, полученные преобразованием (38) из $P$ и $Q$ соответственно. Тогда в силу (39)

$$
\rho\left(z+\gamma_{n}, P, Q\right) \leqslant \rho\left(z, \widehat{P}_{n}, \widehat{Q}_{n}\right) \leqslant \rho(z, P, Q) .
$$

Отсюда с помощью предложения 2 в соответствии с «правилом двух милиционеров» получим

$$
\rho\left(z, \widehat{P}_{n}, \widehat{Q}_{n}\right) \longrightarrow \rho(z, P, Q) \text { при } n \rightarrow \infty .
$$

Для $\widehat{P}_{n}$ и $\widehat{Q}_{n}$ определим $\widehat{S}_{n}$ и $\widehat{T}_{n}$ в соответствии с (15)-(17). Как было доказано выше,

$$
\rho\left(z, \widehat{P}_{n}, \widehat{Q}_{n}\right)=\lim _{y \rightarrow \infty} \widehat{S}_{n}(y)-1=\lim _{y \rightarrow \infty} \widehat{T}_{n}(y)-1 .
$$

При каждом $n \geqslant 1$ индукцией по $j$ с помощью (34), (35) легко показать, что

$$
\widehat{S}_{n}\left(j \gamma_{n}\right) \leqslant S\left(j \gamma_{n}\right), \quad \widehat{T}_{n}\left(j \gamma_{n}\right) \leqslant T\left(j \gamma_{n}\right) \quad \text { при всех целых } j \geqslant 0 .
$$

\section{Следовательно,}

$$
\lim _{y \rightarrow \infty} \widehat{S}_{n}(y) \leqslant \lim _{y \rightarrow \infty} S(y), \quad \lim _{y \rightarrow \infty} \widehat{T}_{n}(y) \leqslant \lim _{y \rightarrow \infty} T(y) \quad \text { при всех } n \geqslant 1 .
$$

Покажем теперь, что выполнено соотношение

$$
\lim _{n \rightarrow \infty} \widehat{S}_{n}(y)=\lim _{y \rightarrow \infty} S(y), \quad \lim _{n \rightarrow \infty} \widehat{T}_{n}(y)=\lim _{y \rightarrow \infty} T(y)
$$

для всех $y \in Y=\left\{j \gamma_{l}: j\right.$ и $l \geqslant 1$ целые $\}$. Имеем $\widehat{S}_{n}(y)=S(y)=\widehat{T}_{n}(y)=$ $T(y)=0$ при $y \leqslant 0$. Предположим, что для всех $y \in Y$ таких, что $y \leqslant k \gamma_{1}$, выполняется (44), и докажем, что тогда (44) выполняется и для $y=k \gamma_{1}+t \gamma_{i}$ при всех цельх $i \geqslant 1$ и $0<t \leqslant 2^{i}$.

Выберем произвольно $\varepsilon>0$ и найдем такое число $n_{0} \geqslant i$, что при всех $n \geqslant n_{0}$

$$
\left|\widehat{S}_{n}\left(k \gamma_{1}+t \gamma_{i}\right)-S\left(k \gamma_{1}+t \gamma_{i}\right)\right|<\varepsilon
$$


В соответствии с (36) имеем

$$
\begin{aligned}
\widehat{S}_{n}\left(k \gamma_{1}+t \gamma_{i}\right)= & \widehat{S}_{n}\left(k \gamma_{1}\right)+P\left(\left[k \gamma_{1}, k \gamma_{1}+t \gamma_{i}\right)\right) \\
& +\max _{0 \leqslant l \leqslant 2^{n-i_{t}}}\left(\widehat{T}_{n}\left(k \gamma_{1}-z+l \gamma_{n}\right)-\widehat{S}_{n}\left(k \gamma_{1}\right)\right. \\
& \left.-P\left(\left[k \gamma_{1}, k \gamma_{1}+l \gamma_{n}\right)\right)\right)^{+} .
\end{aligned}
$$

Заметим теперь, что $T(y)$ - неубывающая, непрерывная слева функция и $\lim _{y \rightarrow \infty} T(y) \leqslant 2$ (из-за того, что $T(y) \leqslant P([0, y))+Q([0, y))$ и $S(y) \leqslant$ $P([0, y))+Q([0, y))$ при всех $y)$. Значит, множество $\nu=\{y: T(y+0)-$ $T(y)>\varepsilon / 6$ или $P(\{y\})>\varepsilon / 6\}$ конечно. Выберем такое целое $n_{1}$, что

$$
\gamma_{n_{1}}<\min \{|a-b|: a \in \nu, b \in \nu, a \neq b\} .
$$

Выберем такое $m \geqslant n_{1}$, что

$$
\begin{aligned}
\sup _{y \in \mathbf{R}}\left\{P\left(\left[y, y+\gamma_{m}\right) \backslash \nu\right)+T\left(y+\gamma_{m}\right)-T(y)\right. \\
\left.-\sum_{a \in \nu \cap\left[y, y+\gamma_{m}\right)}(T(a+0)-T(a))\right\}<\frac{\varepsilon}{2} .
\end{aligned}
$$

Теперь возьмем такое $n_{0} \geqslant m$, что при всех $n \geqslant n_{0}$ выполняется

$$
\begin{aligned}
& 2\left|\widehat{S}_{n}\left(k \gamma_{1}\right)-S\left(k \gamma_{1}\right)\right| \\
& \quad+\max _{0 \leqslant l \leqslant t \cdot 2^{m-i}}\left|\widehat{T}_{n}\left(k \gamma_{1}-z+l \gamma_{m}\right)-T\left(k \gamma_{1}-z+l \gamma_{m}\right)\right|<\frac{\varepsilon}{2} .
\end{aligned}
$$

Ввиду неравенств (47) и (46) при всех $n \geqslant n_{0}, 0<l \leqslant t \cdot 2^{m-i}$ имеем

$$
\begin{aligned}
& \mid \sup _{(l-1) \gamma_{m} \leqslant v \leqslant l \gamma_{m}}\left(T\left(k \gamma_{1}+v-z\right)-S\left(k \gamma_{1}\right)-P\left(\left[k \gamma_{1}, k \gamma_{1}+v\right)\right)\right)^{+} \\
& \quad-\max _{v=(l-1) \gamma_{m}, l \gamma_{m}}\left(T\left(k \gamma_{1}+v-z\right)-S\left(k \gamma_{1}\right)-P\left(\left[k \gamma_{1}, k \gamma_{1}+v\right)\right)\right)^{+} \mid<\frac{\varepsilon}{2} .
\end{aligned}
$$

Значит, при всех $n \geqslant n_{0}$ выполнено

$$
\begin{gathered}
\mid \sup _{0<v \leqslant t \gamma_{i}}\left(T\left(k \gamma_{1}+v-z\right)-S\left(k \gamma_{1}\right)-P\left(\left[k \gamma_{1}, k \gamma_{1}+v\right)\right)\right)^{+} \\
-\max _{0 \leqslant l \leqslant t \cdot 2^{m-i}}\left(T\left(k \gamma_{1}+l \gamma_{m}\right)-S\left(k \gamma_{1}\right)\right. \\
\left.-P\left(\left[k \gamma_{1}, k \gamma_{1}+l \gamma_{m}\right)\right)\right)^{+} \mid<\frac{\varepsilon}{2} .
\end{gathered}
$$

В последнем выражении интервал $\left[k \gamma_{1}, k \gamma_{1}\right)$ означает пустое множество; супремум по $0<v \leqslant t \gamma_{i}$ равен супремуму по $0 \leqslant v \leqslant t \gamma_{i}$, так как 
$S(y) \geqslant T(y-z)$ при всех $y$. Сравнивая выражения (45) и (16), в силу (48) и (49) получим

$$
\widehat{S}_{n}\left(k \gamma_{1}+t \gamma_{i}\right)>S\left(k \gamma_{1}+t \gamma_{i}\right)-\frac{\varepsilon}{2}-\frac{\varepsilon}{2} \quad \text { при всех } n \geqslant n_{0}
$$

и, комбинируя последнюю оценку с (42),

$$
\left|S\left(k \gamma_{1}+t \gamma_{i}\right)-\widehat{S}_{n}\left(k \gamma_{1}+t \gamma_{i}\right)\right|<\varepsilon \text { при всех } n \geqslant n_{0},
$$

что и доказывает (44) при $y=k \gamma_{1}+t \gamma_{i}$, поскольку для $\widehat{T}_{n}$ можно провести аналогичные рассуждения. Следовательно, (44) выполняется при всех $y \in Y$.

Теперь мы можем доказать, что

$$
\begin{aligned}
& \lim _{n \rightarrow \infty} \lim _{y \rightarrow \infty} \widehat{S}_{n}(y)=\lim _{y \rightarrow \infty} S(y), \\
& \lim _{n \rightarrow \infty} \lim _{y \rightarrow \infty} \widehat{T}_{n}(y)=\lim _{y \rightarrow \infty} T(y) .
\end{aligned}
$$

Выберем произвольно $\varepsilon>0$. Возьмем такое число $a$, что $S(a)>$ $\lim _{y \rightarrow \infty} S(y)-\varepsilon / 2$. Выберем $n_{0}$ такое, что $\widehat{S}_{n}(a)>S(a)-\varepsilon / 2$ при всех $n \geqslant$ $n_{0}$. Тогда при всех $n \geqslant n_{0}$ выполнено $\lim _{y \rightarrow \infty} \widehat{S}_{n}(y)>\lim _{y \rightarrow \infty} S(y)-\varepsilon$. Отсюда получим, что $\lim _{n \rightarrow \infty} \lim _{y \rightarrow \infty} \widehat{S}_{n}(y) \geqslant \lim _{y \rightarrow \infty} S(y)$, что совместно с (43) дает $\lim _{n \rightarrow \infty} \lim _{y \rightarrow \infty} \widehat{S}_{n}(y)=\lim _{y \rightarrow \infty} S(y)$. Аналогично $\lim _{n \rightarrow \infty} \lim _{y \rightarrow \infty} \widehat{T}_{n}(y)=\lim _{y \rightarrow \infty} T(y)$. Последние два равенства вместе c (41) и доказывают утверждение теоремы.

З а м е ч а н и е 2. В доказательстве следствия для обоснования сходимости $\rho\left(z, \hat{P}_{n}, \hat{Q}_{n}\right) \longrightarrow \rho(z, P, Q)$ (41) используется непрерывность справа функционала $\rho$ по $z$. Но сходимость (41) можно обосновать и другим способом, вполне аналогичным примененному в доказательстве теоремы 2 в [4]. Выберем такие борелевские вероятностные меры $\mu_{n}$ на $\mathbf{R}^{2}$ с маргиналами $\widehat{P}_{n}$ и $\widehat{Q}_{n}$, что $\mu_{n}\{(a, b):|a-b|>z\}<\rho\left(z, \widehat{P}_{n}, \widehat{Q}_{n}\right)+1 / n$. Тогда можно выбрать подпоследовательность $\left\{\mu_{n_{k}}\right\}_{k}$, слабо сходящуюся $\mathbf{K}$ некоторой борелевской вероятностной мере на $\mathbf{R}^{2}$ с маргиналами $P$ и $Q$. По теореме 1.2 в [6] $\liminf _{k \rightarrow \infty} \mu_{n_{k}}\{(a, b):|a-b|>z\} \geqslant$ $\mu\{(a, b):|a-b|>z\}$. Учитывая, что $\limsup _{n \rightarrow \infty} \mu_{n}\{(a, b):|a-b|>z\} \leqslant$ $\rho(z, P, Q)$ в силу (40), получим $\lim _{k \rightarrow \infty} \mu_{n_{k}}\{(a, b):|a-b|>z\}=\rho(z, P, Q)$, что и влечет $\lim _{k \rightarrow \infty} \rho\left(z, \widehat{P}_{n}, \widehat{Q}_{n}\right)=\rho(z, P, Q)$.

В доказательстве теоремы 2 в [7] используется аппроксимация распределений $P$ и $Q$ распределениями $P_{n}$ и $Q_{n}$ с конечными носителями. При обосновании сходимости $\rho\left(z, P_{n}, Q_{n}\right) \rightarrow \rho(z, P, Q)$ используются рассуждения, аналогичные доказательству теоремы 2 в [4] (а значит, и аналогичные рассуждениям предыдущего абзаца настоящей работы). Однако для того, чтобы эта сходимость имела место, не достаточно только слабой сходимости $P_{n} \rightarrow P, Q_{n} \rightarrow Q$. Необходимо, чтобы также выпол- 
нялось $\lim \sup _{n \rightarrow \infty} \rho\left(z, P_{n}, Q_{n}\right) \leqslant \rho(z, P, Q)$. Приведем пример, поясняющий важность последнего соотношения.

При построении $P_{n}$ и $Q_{n}$ естественно использовать конечные подмножества $V_{1} \subset V_{2} \subset V_{3} \subset \cdots$ некоторой всюду плотной в $U$ последовательности $V$ и строить для каждого $n P_{n}$ и $Q_{n}$ с носителями, сосредоточенными на $V_{n}$. В [7] последовательность $V$ выбирается произвольно. Но, хотя для получения слабой сходимости $P_{n} \rightarrow P, Q_{n} \rightarrow Q$ можно взять произвольную всюду плотную последовательность $V$, для обеспечения сходимости $\rho\left(z, P_{n}, Q_{n}\right) \longrightarrow \rho(z, P, Q)$ последовательность $V$ нельзя выбирать произвольно.

Рассмотрим пространство $U=\{0, z\} \times \mathbf{R}$ с метрикой $|a-b|=$ $\left(\left(a_{1}-b_{1}\right)^{2}+\left(a_{2}-b_{2}\right)^{2}\right)^{1 / 2}$. Пусть $P$ - равномерное распределение на отрезке $\{0\} \times[0,1]$ относительно меры Лебега на этом отрезке, а $Q-$ равномерное распределение на отрезке $\{z\} \times[0,1]$ также относительно меры Лебега на этом отрезке. Очевидно, что $\rho(z, P, Q)=0$, однако если выбрать всюду плотную последовательность $V=\{(0, y): y$ рационально $\} \cup\{(z, y+\sqrt{2}): y$ рационально $\}$, то при любом $n$ будем иметь $\rho\left(z, P_{n}, Q_{n}\right)=1$. И хотя в приведенном примере построение всюду плотной последовательности, ее конечных подмножеств и аппроксимирующих мер $P_{n}$ и $Q_{n}$ таких, что $\rho\left(z, P_{n}, Q_{n}\right) \longrightarrow \rho(z, P, Q)$, не вызывает затруднений, в случае произвольного польского пространства возможность построения аппроксимирующих мер $P_{n}$ и $Q_{n}$ с нужными свойствами, насколько нам известно, пока не доказана.

Доказательство те оремы 1. Существование и единственность функций $S$ и $T$ доказаны в доказательстве предложения 1 . Определим вероятностные меры $P_{L}^{\prime}$ и $Q_{L}^{\prime}$ на $\mathbf{R}$ следующим образом. Положим для любого борелевского $A \subset \mathbf{R}$

$$
\begin{aligned}
P_{L}^{\prime}(A) & =P(A \cap[L, \infty))+I(L-z \in A) \cdot P((-\infty, L)) \\
Q_{L}^{\prime}(A) & =Q(A \cap[L, \infty))+I(L-z \in A) \cdot Q((-\infty, L))
\end{aligned}
$$

где через $I$ обозначена индикаторная функция. Для распределений $P_{L}^{\prime}$ и $Q_{L}^{\prime}$ построим функции $S_{L}^{\prime}$ и $T_{L}^{\prime}$ в соответствии с (15)-(17), взяв константу $K=L-z$. Так как утверждение следствия уже доказано, то

$$
\rho\left(z, P_{L}^{\prime}, Q_{L}^{\prime}\right)=\lim _{y \rightarrow \infty} S_{L}^{\prime}(y)-1=\lim _{y \rightarrow \infty} T_{L}^{\prime}(y)-1
$$

Очевидно, что

$$
\begin{aligned}
& S_{L}^{\prime}(y)=T_{L}^{\prime}(y)=0 \quad \text { при } y \leqslant L-z \\
& S_{L}^{\prime}(y)=P((-\infty, L)), \quad T_{L}^{\prime}(y)=Q((\infty, L)) \quad \text { при } L-z<y \leqslant L .
\end{aligned}
$$


Следовательно, как было установлено в доказательстве предложения 1 (32), для всех $y$ выполнено

$\max \left\{\left|S_{L}^{\prime}(y)-S_{L}(y)\right|,\left|T_{L}^{\prime}(y)-T_{L}(y)\right|\right\} \leqslant \max \{P((-\infty, L)), Q((\infty, L))\}$,

где функции $S_{L}$ и $T_{L}$ определены в (11)-(13). Так как при $L \rightarrow-\infty$ функции $S_{L}$ и $T_{L}$ равномерно сходятся к функциям $S$ и $T$, то функции $S_{L}^{\prime}$ и $T_{L}^{\prime}$ также равномерно сходятся к этим функциям, а значит,

$$
\lim _{L \rightarrow-\infty} \lim _{y \rightarrow \infty} S_{L}^{\prime}(y)=\lim _{y \rightarrow \infty} S(y)=\lim _{L \rightarrow-\infty} \lim _{y \rightarrow \infty} T_{L}^{\prime}(y)=\lim _{y \rightarrow \infty} T(y) .
$$

Из определения функционала $\rho$ следует, что

$$
\rho\left(z, P_{L}^{\prime}, Q_{L}^{\prime}\right) \longrightarrow \rho(z, P, Q) \text { при } L \rightarrow-\infty .
$$

Отсюда ввиду (50) и следует утверждение теоремы 1.

Д ок азат ел в с т в т е о р е м 2. Для произвольного замкнутого множества $H \subset \mathbf{R}$ будем обозначать $H^{+}=H \cap[0, \infty), H^{-}=$ $\{-y: y \in H, y \leqslant 0\}$,

$$
\breve{H}= \begin{cases}H^{+}, & \text {если } \breve{P}\left(H^{+}\right)-\breve{Q}\left(\left(H^{+}\right)^{z}\right) \geqslant \breve{P}\left(H^{-}\right)-\breve{Q}\left(\left(H^{-}\right)^{z}\right), \\ H^{-}, & \text {если } \breve{P}\left(H^{+}\right)-\breve{Q}\left(\left(H^{+}\right)^{z}\right)<\breve{P}\left(H^{-}\right)-\breve{Q}\left(\left(H^{-}\right)^{z}\right),\end{cases}
$$

$\tilde{H}=\left\{y,-y: y \in H^{+}\right\}$. Далее, для произвольного замкнутого $H$ имеем $P(H)-Q\left(H^{z}\right) \leqslant \frac{1}{2}\left(\breve{P}\left(H^{+}\right)+\breve{P}\left(H^{-}\right)\right)-\frac{1}{2}\left(\breve{Q}\left(\left(H^{+}\right)^{z}\right)+\breve{Q}\left(\left(H^{-}\right)^{z}\right)\right) \leqslant$ $\breve{P}(\breve{H})-\breve{Q}\left(\breve{H}^{z}\right)$. Значит, $\sup \left\{P(H)-Q\left(H^{z}\right): H\right.$ замкнутое $\} \leqslant \sup \{\breve{P}(H)-$ $\breve{Q}\left(H^{z}\right): H$ замкнутое $\}$. Также для произвольного замкнутого $H$ имеем $\breve{P}(H)-\breve{Q}\left(H^{z}\right)=P(\widetilde{H})-Q\left(\widetilde{H}^{z}\right)$. А значит, $\sup \left\{P(H)-Q\left(H^{z}\right): H\right.$ замкнутое $\} \geqslant \sup \left\{\breve{P}(H)-\breve{Q}\left(H^{z}\right): H\right.$ замкнутое $\}$. Следовательно, ввиду (4) выполнено

$$
\rho(z, P, Q)=\rho(z, \breve{P}, \breve{Q}) .
$$

3. Примеры. Для обоснования примеров нам потребуется следующеe

3 а м е ч н и е 3 . Если в теореме 1 распределения $P$ и $Q$ непрерывные, то соотношения (6) и (7) эквивалентны выполнению следующих двух условий: при всех $y$

$$
\begin{aligned}
& d S(y)=\max \{d F(y), I(T(y-z) \geqslant S(y)) d T(y-z)\}, \\
& d T(y)=\max \{d G(y), I(S(y-z) \geqslant T(y)) d S(y-z)\} .
\end{aligned}
$$

П р и м е $\mathrm{p} 1$. Пусть $0<s<t$. Обозначим через $P$ и $Q$ показательные распределения с параметрами $s$ и $t$ соответственно. Тогда при Bcex $z \geqslant 0$

$$
\rho(z, P, Q)=e^{-z s t /(t-s)}\left(\left(\frac{s}{t}\right)^{s /(t-s)}-\left(\frac{s}{t}\right)^{t /(t-s)}\right)
$$


Для вычисления последнего выражения воспользуемся следствием. Предположим сначала, что $z>0$. Возьмем константу $K=0$ и построим функции $S$ и $T$ в соответствии с (15) и (51)-(52). Обозначим через $E_{s}$ и $E_{t}$ функции распределения, соответствующие мерам $P$ и $Q$. При всех $y>0$ имеем $E_{s}(y)<E_{t}(y)$. Пусть $y_{1}=\min \left\{y>0: E_{t}(y-z) \geqslant E_{s}(y)\right\}$. Несложно вычислить, что $y_{1}=t z /(t-s)$. Обозначим $y_{2}=(t z+\ln (t / s)) /$ $(t-s)$. Несложно вычислить, что при всех $y, y_{1} \leqslant y<y_{2}$, выполняется $\frac{d}{d y} E_{t}(y-z)>\frac{d}{d y} E_{s}(y)$, а при всех $y>y_{2}$ выполнено $\frac{d}{d y} E_{t}(y-z)<$ $\frac{d}{d y} E_{s}(y)$. Определим функцию $S(y)$ следующим образом:

$$
S(y)=\left\{\begin{array}{lrr}
E_{s}(y) & \text { при } & y \leqslant y_{1}, \\
E_{t}(y-z) & \text { при } & y_{1} \leqslant y \leqslant y_{2} \\
E_{s}(y)+E_{t}\left(y_{2}-z\right)-E_{s}\left(y_{2}\right) & \text { при } & y \geqslant y_{2} .
\end{array}\right.
$$

Очевидно, что $E_{t}(y) \geqslant S(y-z)$ при $y \leqslant y_{2}$. Обозначим $y_{3}=\inf \{y$ : $\left.S(y-z)>E_{t}(y)\right\}$ и определим функцию $T(y)$ следующим образом:

$$
T(y)= \begin{cases}E_{t}(y) & \text { при } y \leqslant y_{3} \\ S(y-z) & \text { при } y \geqslant y_{3}\end{cases}
$$

Несложно проверить, что для построенных так функций $S$ и $T$ выполняются условия (15) и (51)-(52). Очевидно, что

$\lim _{y \rightarrow \infty} S(y)-1=E_{t}\left(y_{2}-z\right)-E_{s}\left(y_{2}\right)=e^{-z s t /(t-s)}\left(\left(\frac{s}{t}\right)^{s /(t-s)}-\left(\frac{s}{t}\right)^{t /(t-s)}\right)$.

Необходимо заметить, что последнее выражение мы вычислили при $z>0$, но по предложению 2 равенство (53) выполняется и при $z=0$.

П р и м р 2. Пусть $0<\sigma<\tau$ и $b$ - некоторые числа. Обозначим через $P$ и $Q$ нормальные распределения с дисперсиями $\sigma^{2}$ и $\tau^{2}$ и математическими ожиданиями $b$ и 0 соответственно. Тогда

$$
\rho(z, P, Q)=\Phi\left(\frac{y_{1}-z}{\tau}\right)-\Phi\left(\frac{y_{1}-b}{\sigma}\right)+\Phi\left(\frac{y_{2}-z-b}{\sigma}\right)-\Phi\left(\frac{y_{2}}{\tau}\right),
$$

где

$$
\begin{aligned}
& y_{1}=\frac{b / \sigma^{2}-z / \tau^{2}-\sqrt{(z-b)^{2} /\left(\sigma^{2} \tau^{2}\right)+2\left(\sigma^{-2}-\tau^{-2}\right) \ln (\tau / \sigma)}}{\sigma^{-2}-\tau^{-2}}, \\
& y_{2}=\frac{(z+b) / \sigma^{2}+\sqrt{(z+b)^{2} /\left(\sigma^{2} \tau^{2}\right)+2\left(\sigma^{-2}-\tau^{-2}\right) \ln (\tau / \sigma)}}{\sigma^{-2}-\tau^{-2}}
\end{aligned}
$$

и $\Phi$ - Функция распределения стандартного нормального закона.

Предположим сначала, что $z>0$, и воспользуемся теоремой 1 . Как и в примере 1 , мы явно построим функции $S$ и $T$, удовлетворяющие 
соотношениям (5) и (51)-(52). Известно, что $F(y)=\Phi((y-b) / \sigma), G(y)=$ $\Phi(y / \tau)$. Обозначим

$$
y_{1}=\min \left\{y: \frac{d}{d y} G(y-z) \leqslant \frac{d}{d y} F(y)\right\} .
$$

Ясно, что нужно положить $S(y)=G(y-z)$ и $T(y)=G(y)$ при $y \leqslant y_{1}$, $S(y)=F(y)+G\left(y_{1}-z\right)-F\left(y_{1}\right)$ при $y_{1} \leqslant y \leqslant w$, где $w=\inf \left\{y>y_{1}: S(y) \neq\right.$ $\left.F(y)+G\left(y_{1}-z\right)-F\left(y_{1}\right)\right\}$. Обозначим $y_{3}=\min \{y: S(y-z) \geqslant G(y)\}$. Тогда $y_{3}<w$ и при всех $y \leqslant y_{3}$ имеем $T(y)=G(y)$. Обозначим также $y_{2}=$ $\min \left\{y>y_{3}: \frac{d}{d y} S(y-z) \leqslant \frac{d}{d y} G(y)\right\}$. Тогда $y_{2}<w$ и при всех $y, y_{3} \leqslant y \leqslant y_{2}$, имеем $T(y)=S(y-z)=F(y-z)+G\left(y_{1}-z\right)-F\left(y_{1}\right)$, а при всех $y \geqslant y_{2}$ имеем $T(y)=G(y)+S\left(y_{2}-z\right)-G\left(y_{2}\right)=G(y)+F\left(y_{2}-z\right)-G\left(y_{2}\right)+G\left(y_{1}-z\right)-$ $F\left(y_{1}\right)$. Значит, $w=\min \left\{y>y_{2}: T(y-z) \geqslant F(y)+G\left(y_{1}-z\right)-F\left(y_{1}\right)\right\}$ и при всех $y \geqslant w$ выполнено $S(y)=T(y-z)$. Таким образом, мы определили функции $S$ и $T$ на всей вещественной прямой. Несложно вычислить, что

$$
\begin{aligned}
& y_{1}=\frac{b / \sigma^{2}-z / \tau^{2}-\sqrt{(z-b)^{2} /\left(\sigma^{2} \tau^{2}\right)+2\left(\sigma^{-2}-\tau^{-2}\right) \ln (\tau / \sigma)}}{\sigma^{-2}-\tau^{-2}}, \\
& y_{2}=\frac{(z+b) / \sigma^{2}+\sqrt{(z+b)^{2} /\left(\sigma^{2} \tau^{2}\right)+2\left(\sigma^{-2}-\tau^{-2}\right) \ln (\tau / \sigma)}}{\sigma^{-2}-\tau^{-2}} .
\end{aligned}
$$

Очевидно, что

$$
\lim _{y \rightarrow \infty} T(y)-1=F\left(y_{2}-z\right)-G\left(y_{2}\right)+G\left(y_{1}-z\right)-F\left(y_{1}\right),
$$

что и доказывает (54) при $z>0$. При $z=0$ равенство (54) выполняется ввиду предложения 2.

4. Построение ближайших случайных величин. Решение задачи Монжа-Канторовича включает в себя также построение ближайmих в смысле (2) случайных величин. Мы построим на одном вероятностном пространстве такие случайные величины $\varphi$ и $\psi$ с распределениями $P$ и $Q$ соответственно, для которых $\mathbf{P}\{|\varphi-\psi|>z\}=\rho(z, P, Q)$. Построение ближайших случайных величин при $z=0$ описано в [5], поэтому всюду далее в этом пункте считаем, что $z>0$.

Пусть $\theta$ - бернуллиевская случайная величина с вероятностью успеха $\rho=\rho(z, P, Q)$ и $\omega-$ равномерно распределенная на отрезке $(0,1)$, случайная величина, не зависящая от $\theta$. Рассмотрим сначала случай $0<\rho<1$. Положим

$$
\begin{aligned}
V(y) & =F(y)-T(y+z)+G(y+z), \\
W(y) & =G(y)-S(y+z)+F(y+z),
\end{aligned}
$$

где функции $S$ и $T$ определены в теореме 1 . Далее мы покажем, что $(1-\rho)^{-1} V(y),(1-\rho)^{-1} W(y), \rho^{-1}(F(y)-V(y)), \rho^{-1}(G(y)-W(y))-$ это 
функции некоторых распределений. Зададим случайные величины $\varphi_{1}$ и $\psi_{1}$ квантильными преобразованиями

$$
\varphi_{1}=\left(\frac{1}{1-\rho} V\right)^{-1}(\omega), \quad \psi_{1}=\left(\frac{1}{1-\rho} W\right)^{-1}(\omega)
$$

Далее будет доказано, что

$$
\left|\varphi_{1}-\psi_{1}\right| \leqslant z \quad \text { п.н. }
$$

Зададим теперь независимые от $\theta$ случайные величины $\varphi_{2}$ и $\psi_{2}$ с распределениями $\rho^{-1}(F-V)$ и $\rho^{-1}(G-W)$ соответственно. Возьмем $\varphi=(1-\theta) \varphi_{1}+\theta \varphi_{2}, \psi=(1-\theta) \psi_{1}+\theta \psi_{2}$. Тогда $\varphi$ и $\psi$ имеют распределения $P$ и $Q$ соответственно, и $\mathbf{P}\{|\varphi-\psi|>z\}=\rho$. В случае $\rho=1$ случайные величины $\varphi$ и $\psi$, с распределениями $P$ и $Q$ соответственно, можно задать произвольно.

В случае $\rho=0$ можно взять квантили $\varphi=F^{-1}(\omega), \psi=G^{-1}(\omega)$. Действительно, в этом случае

$$
S(y) \equiv F(y), \quad T(y) \equiv G(y) .
$$

А значит,

$$
V(y) \equiv F(y), \quad W(y) \equiv G(y), \quad\left|F^{-1}(\omega)-G^{-1}(\omega)\right| \leqslant z \quad \text { п.н. }
$$

Покажем теперь, что функции $(1-\rho)^{-1} V(y),(1-\rho)^{-1} W(y)$, $\rho^{-1}(F(y)-V(y)), \rho^{-1}(G(y)-W(y))$ - это функции распределения. Непрерывность слева и совпадение предела на $+\infty$ с 1 (и на $-\infty$ с 0 ) не вызывает сомнений. Покажем, что эти функции не убывают. Возрастание функций $F(y)-V(y)$ и $G(y)-W(y)$ следует непосредственно из свойств (8) и (9) функций $S$ и $T$.

Покажем, что для любого $w, 0<w<z$, выполнено $V(y+w) \geqslant V(y)$ при всех $y$. Имеем

$$
\begin{aligned}
& V(y+w)-V(y)=F(y+w)-F(y) \\
& \quad-\sup _{0<v \leqslant w}(S(y+v)-T(y+z)-Q([y+z, y+z+v)))^{+} .
\end{aligned}
$$

(В последнем выражении величина $T(y+z+w)$ представлена через $T(y+z)$ по формуле (9).) Последнее выражение неотрицательно, если выполнено

$$
F(y+w)-F(y)-S(y+w)+T(y+z) \geqslant 0 .
$$

Выразим теперь $S(y+w)$ через $S(y)$ по формуле (8). Имеем

$$
-S(y)-\sup _{0<v \leqslant w}(T(y-z+v)-S(y)-P([y, y+v)))^{+}+T(y+z) \geqslant 0 .
$$


Для справедливости последнего неравенства достаточно, чтобы выполнялось $-S(y)-T(y-z+w)+S(y)+T(y+z) \geqslant 0$. Последнее соотношение всегда верно при $0<w<z$. Следовательно, функция $V(y)$ не убывает. Аналогично не убывает и $W(y)$.

Докажем теперь (55). Для этого достаточно показать, что

$$
W(y-z) \leqslant V(y) \leqslant W(y+z) \text { при всех } y .
$$

Покажем, что при всех $y$ выполнено $V(y) \geqslant W(y-z)$. Имеем

$$
V(y)-W(y-z)=G(y+z)-G(y-z)-T(y+z)+S(y) .
$$

Выразим $T(y+z)$ через $T(y-z)$ по формуле (9):

$$
\begin{aligned}
V(y)-W(y-z) & \geqslant S(y)-T(y-z)-\sup _{0<v \leqslant 2 z}(S(y-2 z+v)-T(y-z))^{+} \\
& \geqslant S(y)-T(y-z)-S(y)+T(y-z)=0 .
\end{aligned}
$$

Значит, $V(y) \geqslant W(y-z)$ при всех $y$. Аналогично, $V(y) \leqslant W(y+z)$ при всех $y$. Следовательно, имеет место (56).

5. Пуассоновская аппроксимация биномиального распределения. Обозначим через $Q$ биномиальное распределение с параметрами $n, p$, а через $P$ - пуассоновское распределение с параметром $\lambda=n p$. В этом пункте оценивается величина $\rho(z, P, Q)$.

Один из первых результатов, связанных с данной проблемой, был получен Ю.В. Прохоровым в 1953 г. (см. [9]). Им было доказано, что $\rho(0, P, Q) \leqslant c p$, где $c-$ абсолютная постоянная. Позже Л. Ле Кам установил (см. $[10])$, что также выполнено $\rho(0, P, Q) \leqslant n p^{2}$. Комбинируя эти два неравенства, получим

$$
\rho(0, P, Q) \leqslant \min \left\{c p, n p^{2}\right\} .
$$

А. Барбур и П. Холл в [11] показали, что имеет место аналогичная оценка снизу.

В работах [12]-[15], [8] при исследовании потраекторной близости процесса частных сумм и соответствуюшего пуассоновского процесса были получены, как частные случаи, оценки расстояния $\rho(z, P, Q)$ для произвольного $z$. Наиболее сильные оценки потраекторной близости содержатся в работе И.С. Борисова [8]. В частности, для $n$-х сумм выполняется

$$
\rho(z, P, Q) \leqslant \begin{cases}\left(n p^{2}\right)^{\lfloor z\rfloor+1} \exp \left\{-C_{1} z \ln \ln (z+2)+C_{2}\right\}, & \text { если } n p \geqslant 1, \\ n p^{\lfloor z\rfloor+2} \exp \left\{-C_{3} z+C_{4}\right\}, & \text { если } n p \leqslant 1,\end{cases}
$$

где $C_{1}, C_{2}, C_{3}, C_{4}-$ абсолютные положительные постоянные. 
Однако для $n$-х сумм эти оценки уже не являются оптимальными. Так, П. Майор в [12] доказал, что для $p \leqslant n^{-2 / 3}$ имеет место неравенство

$$
\rho(C, P, Q)<K \exp \left(-\frac{1}{8} \sqrt{n} \ln n\right),
$$

где $C, K$ - некоторые абсолютные постоянные.

С помощью теоремы 1 в [16] доказан следующий результат.

Теорема 4. Пусть $p \leqslant 1 / 2 u z \geqslant 1$ - челое. Тогда

$$
\begin{aligned}
& \rho(z, P, Q)<\frac{7}{3} \exp \left\{-\frac{1}{27} \frac{z^{2}}{n p^{3}}\right\} n p u z \leqslant n p^{2}, \\
& \rho(z, P, Q)<\exp \left\{-\frac{1}{15} n p\right\} \quad \text { npu } z \geqslant n p^{2} \text {, } \\
& \rho(z, P, Q)<\exp \left\{-\frac{1}{4} \sqrt{n z\left(\ln \frac{z}{n p^{2}}-2\right)^{3}}\right\} \\
& n p u e^{4} n p^{2} \leqslant z \leqslant \frac{n(1-p)}{\ln (1 / p)}, \\
& \rho(z, P, Q)=P([n+z+1, \infty))<\exp \left\{-(n+z) \ln \frac{n+z}{e n p}\right\} \\
& n p u z \geqslant \frac{n(1-p)}{\ln (1 / p)} \text {. }
\end{aligned}
$$

Кроме того,

$$
\begin{aligned}
& \rho(z, P, Q)>\exp \left\{-4 \sqrt{n z\left(\ln \frac{z}{n p^{2}}+4\right)^{3}}\right\}, \\
& \text { если } p \leqslant \frac{1}{10} u n p^{2} \leqslant z \leqslant \frac{n}{10 \ln (1 / p)} .
\end{aligned}
$$

3 а м е ч а н и е 4. Неравенство (61) показывает, что в некотором диапазоне значений $n, p, z$ оценки (58), (59) имеют неулучшаемый порядок логарифмической асимптотики. Оценка (60) также точна в этом смысле, так как

$$
\begin{aligned}
& P([n+z+1, \infty)) \\
& \quad=\frac{\theta}{\sqrt{2 \pi(n+z+1)}} \exp \left\{-(n+z+1) \ln \frac{n+z+1}{e n p}-n p\right\} .
\end{aligned}
$$

где $e^{-1 / 12(n+z+2)}<\theta<(1-p)^{-1} e^{-1 / 12(n+z+1)}$ (см. [16]).

В заключение сравним утверждение теоремы с оценкой (57).

1. В отличие от результата П. Майора, теорема 4 содержит оценки расстояния $\rho(t, P, Q)$ для всех $t \geqslant 1$. 
2. Для того чтобы применение теоремы 4 имело смысл, достаточно, чтобы $n p^{3} \rightarrow 0$, в то время как для выполнения неравенства (57) необходимо, чтобы $n p^{3 / 2} \leqslant 1$.

3. Для фиксированного $z$ равномерно по всем $p \leqslant n^{-2 / 3}$ в силу неравенств (59), (60) имеем

$$
\rho(z, P, Q)<K_{1} \exp \left\{-K_{2} \sqrt{n(\ln n)^{3}}\right\},
$$

где $K_{1}$ и $K_{2}$ - легко вычисляемые абсолютные положительные постоянные. Следовательно, при фиксированном $z$ и всех $p \leqslant n^{-2 / 3}$ оценка теоремы 4 имеет большую, чем в неравенстве (57), скорость убывания.

\section{СПИСОК ЛИТЕРАТУРЫ}

1. Каяторович Л. В. О перемещении масс. - Докл. АН СССР, 1942, т. 37, № 7-8, c. 227-229.

2. Каяторович Л. В. Об одной проблеме Монжа. - Успехи матем. наук, 1948, т. 3, B. 2, c. 225-226.

3. Рачев C. Т. Задача Монжа-Канторовича о перемещении масс и ее применения в стохастике. - Теория вероятн. и ее примен., 1984, т. 29, в. 4, с. 625-653.

4. Dudley R. M. Distances of probability measures and random variables. - Ann. Math. Statist., 1968, v. 39, № 5, p. 1563-1572.

5. Добрушия $Р$. Л. Задание системы случайных величин при помощи условных распределений. - Теория вероятн. и ее примен., 1970, т. 15, в. 3, с. 469-497.

6. Прохоров Ю. В. Сходимость случайных процессов и предельные теоремы теории вероятностей. - Теория вероятн. и ее примен., 1956, т. 1, в. 2, с. 177-238.

7. Schay $G$. Nearest random variables with given distributions. - Ann. Probab., 1974, v. 2 , № 1 , p. $163-166$.

8. Борисов И. С. Пуассоновская аппроксимация процесса частных сумм в банаховых пространствах. -- Сиб. матем. журн., 1996, т. 37, в. 4, с. 723-731.

9. Прохоров Ю.В. Асимптотическое поведение биномиального распределения. Успехи матем. наук, 1953, в. 8, № 3, с. 135-142.

10. Le $\operatorname{Cam} L$. Remarques sur le théorème limite central dans les espaces localement convexes. - Les Probabilitiés sur les Structures Algébriques (Actes Colloque Internat., v. 186). Paris: C.N.R.S., 1970, p. 233-249.

11. Barbour A. D., Hall P. On the rate of Poisson convergence. - Math. Proc. Cambridge Philos. Soc., 1984, v. 95, p. 473-480.

12. Major $P$. A note on the approximation of the uniform empirical process. - Ann. Probab., 1990, v. 18, № 1, p. 129-139.

13. Horváth $L$. A note on the rate of Poisson approximation of empirical processes. Ann. Probab., 1990, v. 18, № 2, p. 724-726.

14. Borisov I. S. Strong Poisson and mixed approximations of sums of independent random values in Banach spaces. - Siberian Adv. Math., 1993, v. 3, № 2, p. 1-13.

15. Adell J.A., de la Cal J. Coupling methods in Poisson approximation of binomial processes. Preprint. Zaragoza: Universidad de Zaragoza, 1994.

16. Рузаякия П. С. О пуассоновской аппроксимации биномиального распределения. - Сиб. матем. журн., 2001, т. 42, в. 2, с. 414-424. 